SCIREA Journal of Clinical Medicine

ISSN: 2706-8870

http://www.scirea.org/journal/CM

December 28, 2021

SCIREA

Volume 6, Issue 6, December 2021

https://doi.org/10.54647/cm32745

\title{
Outcome of COVID 19 in hemodialysis patients: single center experience
}

Salwa Mahmoud Elwasif *, Mohamed Osama Megahed, Muhammed Ahmed Elhadedy, Ahmed Farouk Donia, Aymen Fathi Refaie

Nephrology and renal transplantation unit, Urology and Nephrology Center, Mansoura University, Egypt

* Corresponding author:

Salwa Mahmoud Elwasif, MD, Urology and Nephrology Center, Mansoura University Mobile: 00201003700562 Email: drsalwa22000@yahoo.com

\begin{abstract}
:
Background: Generally end-stage kidney disease (ESKD) patients are more prone to infection, especially patients maintained on Hemodialysis because of frequent attendance to hemodialysis units. Due to their impaired immune response, patients with ESRD are more likely to develop severe complications of any infectious disease.
\end{abstract}

Objectives: to study and analyze all available patients' data and find out the correctable predictors for mortality to overcome them.

Patients and methods: Our study is a retrospective cohort one, done in urology and nephrology center, Mansoura University, Egypt. We have ninety-eight patients scheduled for hemodialysis; most of them have three sessions per week. 
We studied the outcome of COVID-19 in ESKD patients receiving hemodialysis as regard their fate. Patients' Data during infection were tabulated and analyzed searching for significant predictors for fruitful outcome.

Results: Before COVID pandemic, we had ninety eight patients receiving hemodialysis service in our out-patient clinic. From whom, there were seventeen cases (17.3\%) developed SARS-COV disease. Six of them (6.12\%) lost their life due to sever complications. Old age, diabetes mellitus, low flux dialyzer and interdialytic weights gain more than 3 liters are predictors for mortality.

Conclusion: Patients' compliance for Intradialytic weights gain, high flux dialyzer, restricts control of blood sugar and treatments of comorbidities are essential for better outcome of COVID among hemodialysis patients.

Keyword: COVID-19, hemodialysis, outcome

\section{Introduction:}

COVID-19 is highly contagious disease that appears first at the end of 2019. World health organization recalls it as pandemic in March 2020. All countries closed their borders and many of them do curfew for their citizens. ${ }^{(\mathbf{1})}$ Hospital-based Hemodialysis patients need to visit hospital regularly and that make them more prone to infection either nosocomial or during transportation ${ }^{(2)}$.

Patients with ESKD are more likely to develop severe complications of coronavirus disease because of the high burden of comorbidities, and their impaired immune response ${ }^{(3)}$. Social distance and adopting infection control strategies can lower the incidence of infection but the outcome differs among patients. Therefore, we conducted this study in order to assess prevalence and mortality predictors of COVID-19 infection among a cohort of Egyptian hemodialysis patients.

\section{Patient and methods:}

Our study is a retrospective cohort study done in Urology and Nephrology center, Mansoura University. Among ninety eight patients receiving scheduled hemodialysis in our out-patient 
clinic, there are seventeen patients developed COVID-19 during period between March 2020 and end of January 2021. Any patients suffered from fever, respiratory symptoms and/or diarrhea was subjected to chest computerized tomography (CT) without contrast and complete blood count. We use CORAD classification for assessment of chest C.T. Patient with leucopenia or lymphopenia and/or suspicious CT was subjected to SARS-COV 2 universal reverse transcriptase-Polymerase Chain Reaction (PCR). All positive PCR patients are included in our study. Average interdialytic weight gain was measured by calculated mean during a month of getting infected. Kt/V Daugirdas was calculated online (4).

All patients received treatment according to updated Egyptian ministry of health protocol ${ }^{(5)}$. They were dialyzed by intermittent hemodialysis, three times per week. We defined the cure by one who has no symptoms and negative SARS-COV PCR. We classified COVID--infected patients into two groups according to their survival. Group 1 included patients who survived and group 2 constituted patients who died after COVID 19 infection.

Continuous data was compared using Student $t$ test and Mann-Whitney test for normal and NON-normal distributed data respectively. Categorical data was compared using chi-square test. Inferential statistics were performed by IBM SPSS (version 19) software program. A pvalue $<0.05$ was considered significant.

\section{Results:}

Among ninety eight patients receiving hemodialysis service in our out-patient clinic, there were seventeen cases (17.3\%) developed SARS-COV disease. Six of them $(6.12 \%)$ lost their life due to severe complications.

We analyzed all patients' information searching for predictors for survival. (Table 1)

Table 1: patients' data and laboratory results of both groups.

$\begin{array}{lccc} & \text { Group1(n=11) } & \text { Group2 (n=6) } & \text { P value } \\ \text { Age: Mean } \pm \text { SD } & 40.3 \pm 16.8 & 59.5 \pm 13.4 & 0.001 \\ \text { Sex (M:F) } & (9: 2) & (4: 2) & 0244 \\ \text { Original kidney disease: } & & & \\ \text { Unknown } & 6 / 11 & 3 / 6 & 0.850 \\ \text { Diabetic nephropathy } & 1 / 11 & 2 / 6 & \\ \text { Urinary track abnormalities } & 4 / 11 & 1 / 3 & 0.203 \\ \text { Smoker } & 6 / 11 & 2 / 6 & \end{array}$


Influenza vaccine

BMI: Mean \pm SD

Duration on dialysis in years: median (range)

$\mathrm{Kt} / \mathrm{V}:$ Mean $\pm \mathrm{SD}$

High flux/low flux dialyzer

Dialyzer surface area

Hypertension

Diabetes

Intradialytic weight gain: $\quad$ Mean \pm SD

Ischemic cardiomyopathy

Chronic liver disease

Hemoglobin in gm/dl: Mean \pm SD

i-PTH in $\mathrm{pg} / \mathrm{ml}$ : median (range)

Pre-dialysis sodium in $\mathrm{Mmol} / \mathrm{L}$

Post-dialysis sodium in $\mathrm{Mmol} / \mathrm{L}$

Pre-dialysis potassium in $\mathrm{Mmol} / \mathrm{L}$

Post-dialysis potassium in Mmol/L

Serum calcium in $\mathrm{mg} / \mathrm{dl}$

Serum phosphorus in $\mathrm{mg} / \mathrm{dl}$

Serum Albumin in gm/dl
$2.6 \pm 0.84$

$6 / 11$

$2 / 11$

$10.5 \pm 0.8$

$582(92.7-2000)$

$135.09 \pm 2.948$

$136.09 \pm 2.982$

$5.336 \pm 0.5662$

$3.536 \pm 0.4843$

$9.14 \pm 0.75$

$4.59 \pm 1.07$

$3.59 \pm 0.61$
$3 / 6$

0.020

$25.36 \pm 5.85$

0.449

$22.97(11.9-37.6)$

0.225

$1.2 \pm 0.187$

0.266

$3 / 3$

0.020

$1.66 \pm 0.516$

0.908

$2 / 6$

0.25

$2 / 6$

0.02

$3.9 \pm 1.5$

0.03

$4 / 6$

0.61

$1 / 6$

0.88

$11.1 \pm 0.6$

0.139

$765(51-2000)$

0.73

$133.33 \pm 3.830$

0.306

$134.33 \pm 2.338$

0.233

$4.650 \pm 0.9834$

0.085

$3.550 \pm 0.5788$

0.959

$9.56 \pm 0.73$

0.408

$3.86 \pm 0.32$

0.281

$3.05 \pm 0.44$

0.079

Table2: Patients' symptoms and CO-RAD stage in both group.

\section{Symptoms}

dyspnea

GIT symptoms

Loss of smell and taste

Loss of lean body weight

CO-RAD stage:

Stage 3

Stage 4

Stage 5
$1 / 11$

$3 / 11$

$3 / 11$

$3 / 11$

$10 / 11$

$3 / 11$

$7 / 11$

$\begin{array}{cc}6 / 6 & 0.01 \\ 2 / 6 & 0.787 \\ 6 / 6 & 0.98 \\ 4 / 6 & 0.115\end{array}$

$1 / 6$

$0 / 6$

$5 / 6$ 
As regard SARS-COV symptoms, Dyspnea was more in mortality group while diarrhea and vomiting were statistically insignificant. We observe loss of lean body weight up to 5 kilograms in four patients in mortality group comparing to only three cases in survivors.

\section{Discussion:}

In urology and nephrology center (UNC) - Mansoura University, Egypt, there was a hemodialysis unit serving ninety eight patients in March 2020. During COVID pandemic, $17.3 \%$ of our patients developed infection despite adopting preventive measures. The incidence rate of COVID disease in UNC was lower than in patients in Paris, France (19\%). This result is comparable with other reports in Wuhan and Brescia (Italy) has reported an incidence rate in hemodialysis patients of $15 \%$ and $16 \%$ respectively. ${ }^{(6)}$

There was $54.5 \%$ of survivor group versus $33.3 \%$ of mortality group smoke cigarettes. It does not mean that smoking is protective, Perhaps This due to small group population or other confounders in mortality group. ${ }^{(7)}$

The presence of diabetes was associated with mortality this was consistent with the Chinese report that clarify the role of diabetes in up grading of angiotensin converting enzyme 2 receptor that in turn rises the severity of COVID. ${ }^{(8)}$

According to our local policy of vaccination, we gave injectable inactive influenza vaccine to all patients after informed consent that explains the pons and cons of being vaccinated. In survivors group there were nine patients received vaccine $(81.8 \%)$ while in mortality group only $3(50 \%)$. It means that influenza vaccine may protect against sever form of the disease. In spite of being two different viruses, there are many similar characters. ${ }^{(9)}$ some authors discuss the protective role of influenza vaccine against both infection and severity of COVID 19. ${ }^{(10)}$

We observe that Interdialytic weight gain above 3 kilograms per session was associated with mortality among COVID 19 patients (odds ratio= 3.6). This may be due to associated pulmonary edema \pm hemodynamic instability during hemodialysis session. For the best of our knowledge, no one correlate intradialytic weight gain with mortality from COVID 19. Some authors link the fatality with appearance of leaky lung as a part of ARDS. ${ }^{(11,12)}$ 
Insignificant $\mathrm{p}$ value in assessment of CO-RAD denoting that the severity of CT finding does not associated with poor prognosis so, we consider CORAD as a diagnostic not a prognostic tool. This in agreement with Penha et al who concluded the same opinion. ${ }^{(13)}$

Patients with Old age have more risk for mortality due to COVID 19; this may be due to associated comorbidity or even immune senesce. We found the same in hemodialysis patients. While Goicoechea and his coworkers found the adverse. ${ }^{(6)}$

High flux dialyzer is better than low flux one in long stand management of hemodialysis patient especially during infection however it has no effect in pulmonary function test ${ }^{(14)}$ In our study, we find that high flux dialyzer was used more in survival group.

\section{Conclusion:}

During COVID pandemic, Hemodialysis patients need frequent monitoring and management of comorbidities to secure their life and pass safely.

Patient compliance for intradialytic weight gain, high flux dialyzer and restrict control of plasma glucose look like safety valves for better outcome.

\section{Statement of Ethics:}

The idea and the protocol of the study were discussed thoroughly in scientific committee of Mansoura urology and nephrology center applying the principle of Helsinki Declaration.

\section{Author Contributions:}

- The idea of the study: Dr. Salwa Elwasif and prof. Ahmed Donia

- $\quad$ Collection of patients data: Dr. Mohamed osama Megahed

- $\quad$ Analysis of data via SPSS : dr. Muhamed Ahmed Elhadidi

- Writing: Dr. Salwa Mahmoud Elwasif

- Revision of both statistical analysis of data and writing: prof. Ahmed Farouk Donia and Prof. Aymen Fathi Refaie 


\section{Reference:}

[1] Abolfotouh MA, Almutairi AF, Banimustafa A, Hagras SA, Al Jeraisy M. Behavior Responses and Attitude of the Public to COVID-19 Pandemic During Movement Restrictions in Saudi Arabia. International journal of general medicine. 2021;14:741-53.

[2] Medjeral-Thomas NR, Thomson T, Ashby D, Muthusamy A, Nevin M, Duncan N, et al. Cohort Study of Outpatient Hemodialysis Management Strategies for COVID-19 in North-West London. Kidney international reports. 2020;5(11):2055-65.

[3] Akbarialiabad H, Kavousi S, Ghahramani A, Bastani B, Ghahramani N. COVID-19 and maintenance hemodialysis: a systematic scoping review of practice guidelines. BMC Nephrology. 2020;21(1):470.

[4] QXMD Cb. Kt/V Daugirdas: Quantify hemodialysis adequancy 2015, July 2 [Available from: https://qxmd.com/calculate/calculator_128/kt-v-daugirdas.

[5] health emo. protocol of management of covid. 2020.

[6] Goicoechea M, Sánchez Cámara LA, Macías N, Muñoz de Morales A, Rojas Á G, Bascuñana A, et al. COVID-19: clinical course and outcomes of 36 hemodialysis patients in Spain. Kidney international. 2020;98(1):27-34.

[7] Rossato M, Di Vincenzo A. Cigarette smoking and COVID-19. Pulmonology. 2021.

[8] Ma Y, Diao B, Lv X, Liang W, Zhu J, Liu L, et al. COVID-19 in hemodialysis (HD) patients: Report from one HD center in Wuhan, China. medRxiv. 2020:2020.02.24.20027201.

[9] Flerlage T, Boyd DF, Meliopoulos V, Thomas PG, Schultz-Cherry S. Influenza virus and SARS-CoV-2: pathogenesis and host responses in the respiratory tract. Nature reviews Microbiology. 2021:1-17.

[10] Conlon A, Ashur C, Washer L, Eagle KA, Hofmann Bowman MA. Impact of the influenza vaccine on COVID-19 infection rates and severity. American Journal of Infection Control. 2021.

[11] Alharthy A, Faqihi F, Memish ZA, Karakitsos D. Lung Injury in COVID-19-An Emerging Hypothesis. ACS chemical neuroscience. 2020;11(15):2156-8.

[12] U.R A, Verma K. Pulmonary Edema in COVID19-A Neural Hypothesis. ACS chemical neuroscience. 2020;11(14):2048-50.

[13] Diana Penha , Fernando Matos, Colin Monaghan, Luís Taborda-Barata, Klaus Irion, Edson Marchiori. CO-RADS: Coronavirus Classification Review. Cardiopulmonary Imaging Review Article. 2021;11(9). 
[14] Momeni A, Rouhi H, Kiani G, Amiri M. Effects of High-Flux versus Low-Flux Membranes on Pulmonary Function Tests in Hemodialysis Patients. Tanaffos. 2013;12(1):31-5. 